\title{
Management of abdominal wall defects - case series
}

\author{
Adrian Pelinaru', Andreea Grosu-Bularda', Carmen Mocanu1, Adrian Frunza1,2, \\ Razvan Nicolae Teodoreanu ${ }^{1,2}$, Ionut Cosmin Olteanu', Victor Grama ${ }^{3}$, \\ Florin Mihail Iordache ${ }^{2,3}$, Serban Arghir Popescu ${ }^{1,2}$, Ioan Lascar ${ }^{1,2}$ \\ ${ }^{1}$ Clinic of Plastic Surgery, Aesthetic and Reconstructive Microsurgery, \\ Clinical Emergency Hospital, Bucharest, Romania \\ 2 "Carol Davila“ University of Medicine and Pharmacy, Bucharest, Romania \\ ${ }^{3}$ Clinic of General Surgery I, Clinical Emergency Hospital, Bucharest, Romania
}

\begin{abstract}
Treating complex abdominal wall defects is challenging for the reconstructive surgeon as there are many possible complications and high reccurence rates. To date, there is no consensus on a definition of complex abdominal wall defects, as the level of complexity can be different from case to case. Thorough knowledge of the etiology of defects and relevant anatomy, adequate management during hospitalization and in postoperative period are key elements in obtaining optimal functional results. Although the etiology of abdominal wall defects is varied, reconstructive goals are well established, including recovery of structural integrity to ensure visceral coverage and maintenance of intraabdominal pressure, prevention of hernia formation, if possible with an aesthetic pleasant result; long hospitalizations increasing morbidity and costs should be avoided. Choosing the right therapeutic strategy may be very demanding, because there is no standard treating protocol to guide the management of these patients.. We present, through five clinical cases, our experience with managing abdominal wall defects having in mind the general reconstructive priciples and trying to individualize the treatment for each of them.
\end{abstract}

Keywords: abdominal wall defect, reconstruction, risk factors

\section{BACKGROUND}

The abdominal wall pathology is really challenging for the reconstructive surgeon as there are many possible complications and high reccurence rates [1]. To date, there is no consensus on a definition of complex abdominal wall defects, as the level of complexity can be different from case to case [2]. The complex abdominal wall defects may vary both in surface and depth, involving different layers of the abdominal wall, from simple skin loss to complicated and infected wounds that lack of viable local muscles. Sometimes, the severity is just so high that primary fascial closure is impossible and visceral organs are involved, even with loss of domain [3]. The most common causes that lead to such defects are abdominal injury (including burn injuries) or politrauma, local infections such as necrotizing fasceitis and tumoral resection possibily followed by radiation necrosis [4].

Usually the patients have different clinical presentations and the treatment has to be individualized in order to have optimal results. There are different reconstructive options that were studied and improved over time and choosing the suitable one has to follow the reconstructive ladder [3,5] (fig. 1).

An important concept of abdominal wall reconstruction is optimizing the risk factors in order to lower the risk of wound complications. Currently, Ventral Hernia Working Group grading system is the most used one in order to predict surgical site occurences [6] (fig. 2). 


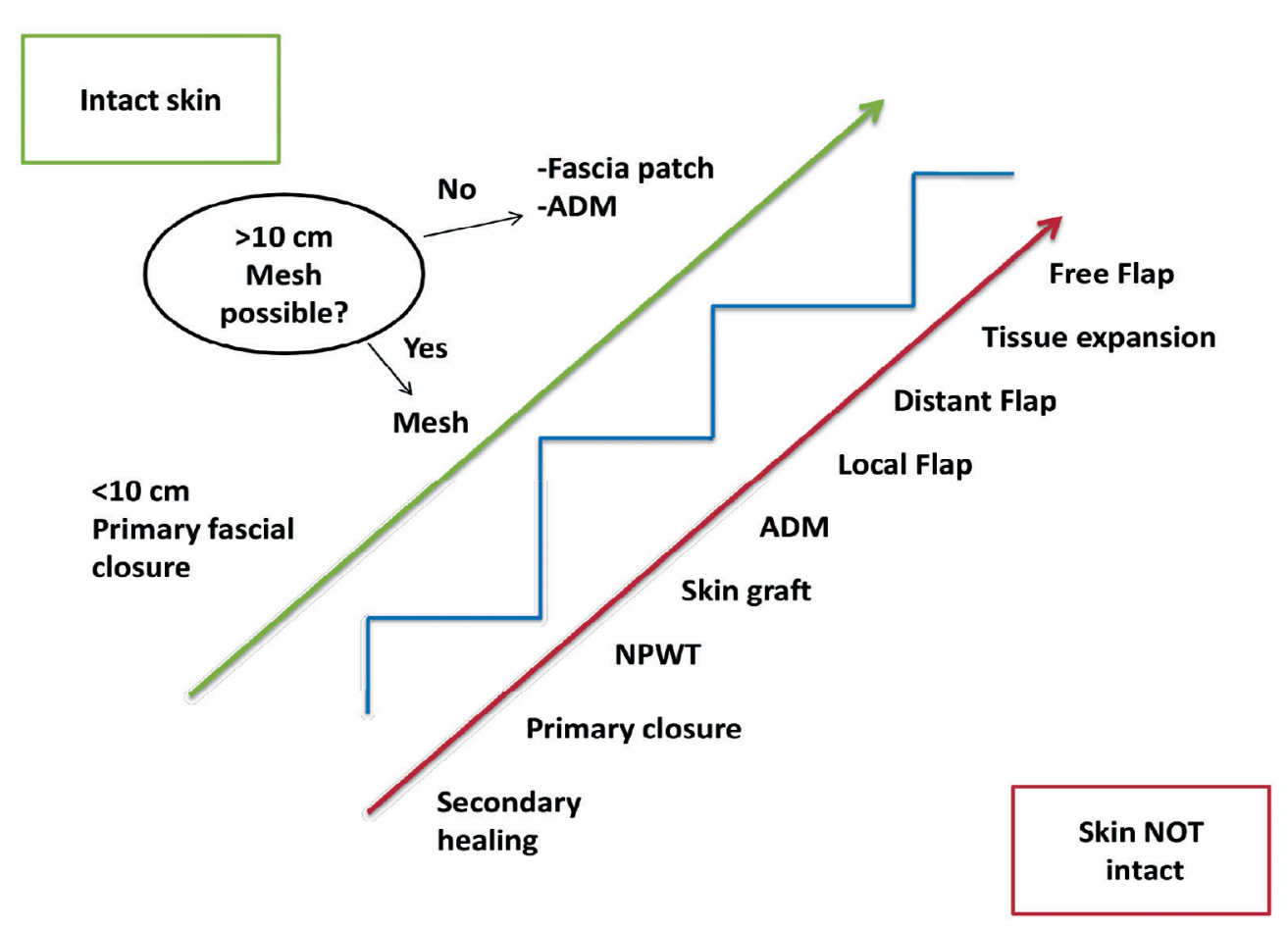

FIGURE 1. The reconstructive ladder of abdominal wall reconstruction
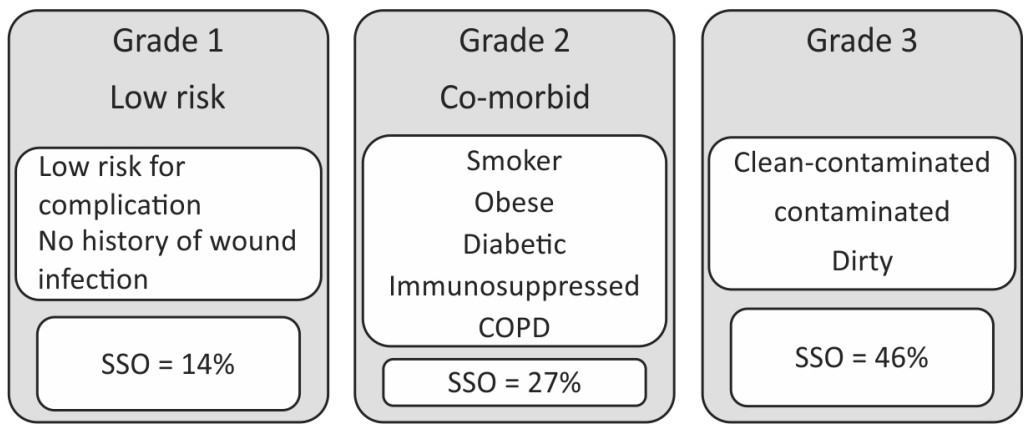

FIGURE 2. The VHWG grading system for Surgical Site Occurences (SSO - dehiscence, seroma, infection, fistula)

\section{METHOD}

We have selected five cases that are relevant for the pathology studied. We present the evolution of the patients in a dynamic way during hospitalization, the complications that occurred and therapeutic principles that were applied.

The patients were admitted in the Bucharest Clinical Emergency Hospital, in different departments such as Plastic Surgery, General Surgery and the Critical Care Burn Unit and required complex, interdisciplinary care. All cases show the management of complex abdominal wall defects correlated to the aforementioned treatment principles.

\section{CLINICAL CASES PRESENTATION}

\section{Case 1}

A 64 y.o. woman, known with obesity and hypertension, with multiple previous umbilical hernia repair attempts, presented to the ER with altered general condition and abdominal wall wound infection after last surgical intervention. The clinical examination revealed an increase of the abdominal volume, with a large eventration on the midline, complicated with enterocutaneous fistula, local soft tissue necrosis and abdominal wall cellulitis. The abdominal radiography showed multiple hydroaer- 
ic levels in the mid and lower abdomen and the ultrasound examination did not reveal any fluid collection. The patient was taken to the operating room and a laparotomy was performed, with partial bowel resection and placement of a right ileostomy. The enterocutaneous fistula was resected, along with all infected adiacent tissue and antibiotic treatment was started. The following days, multiple soft tissue resections were performed resulting in a large abdominal wall defect, until the wound was clean enough to allow a direct closure. The evolution was slowly favorable, and the patient was dischared after 48 days (figure 3 ).
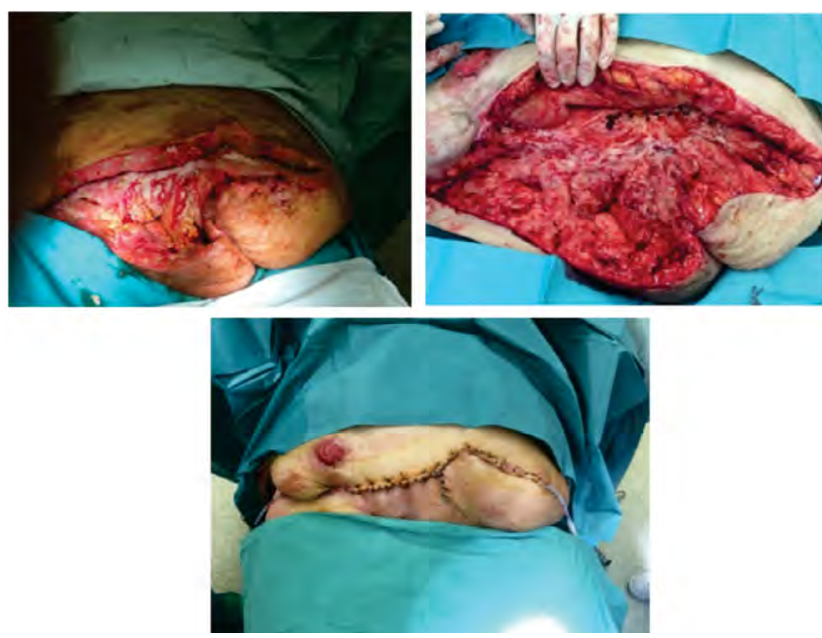

FIGURE 3. Case 1

\section{Case 2}

A 62 years old female, with obesity and diabetes type 2, who suffered mutliple previous abdominal surgery (total hysterectomy, appendicetomy, recto-sigmoid resection with left permanent ileostomy) was admitted to the hospital for a large median eventration complicated with extensive soft tissue infection and multiple enterocutaneous fistulas around the umbilicus. CT findings revealed a 20 $\mathrm{cm}$ eventration, secondary dissemination lesions of the inferior abdominal wall and multiple hydroaeric levels. An immediate surgical intervention was performed and after the resection of all fistulas an infected polypropylene mesh was found and removed. After segmentary bowel resection and infected soft tissue removal, the chosen solution for defect coverage was a vacuum assisted therapy with antibiotic instilation. The patient was admitted to the Intensive Care Unit postoperatory. After 14 days of continous vacuum dressing, the wound was prepared for skin grafting, but despite reanimation, the patient suffered a cardiac arrest and died (figure 4).
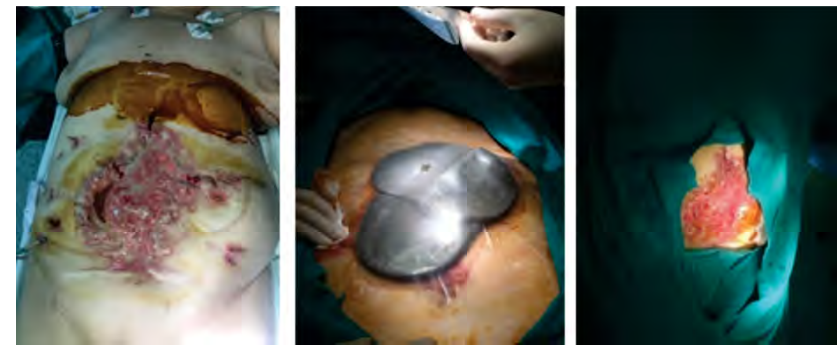

FIGURE 4. Case 2

\section{Case 3}

A 30 years old man was brought by ambulance to the ER after suffering o polytrauma in car crash accident. The injuries included visceral cranial trauma, post-traumatic eventration in the right upper and lower quadrant, rupture of the inferior vena cava, with retroperitoneal hematoma and rupture of transverse colon with haemoperitoneum. Due to traumatic shock and severe hemorrage, an emergency intervention was performed and the vena cava was repaired, all fluid collection drained, a miorrhaphy of the right rectus abdominis muscle performed and all necrotic tissue removed. Postoperatory, the general condition improved, but after 7 days the patient accused a severe pain in the right lumbar area and a CT scan revealed a persistent retroperitoneal hematoma and thrombosis of the right renal artery. A second intervention was performed to remove the right kidney and resect the soft tissue that continued to be necrotic on the midline of the abdominal wall and the right inguinal area. A third surgical procedure was necessary to excise the damaged soft tissue of the abdominal wall and the abdomen was left open and covered with dressings for a planned reconstruction. At 14 days after admission, the patient died of cardiac arrest (figure 5).
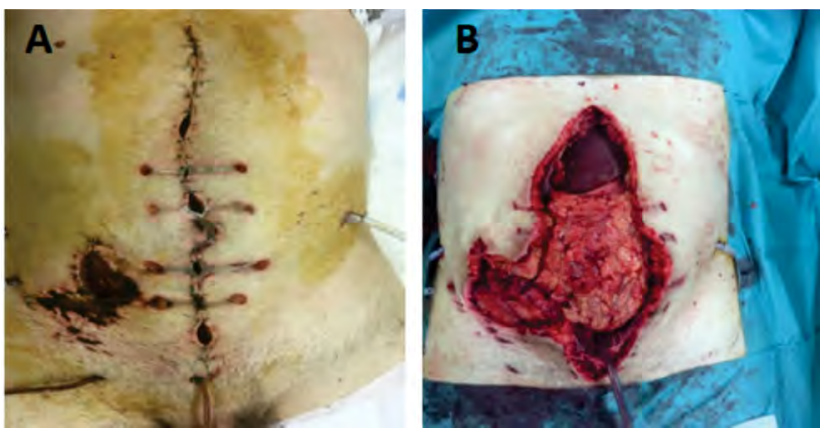

FIGURE 5. Case 3-A. Abdominal wall necrosis; $B$. Aspect after extensive resection resulting in a complex defect

\section{Case 4}

A 56 years old male patient, known with pulmonary carcinoma and disseminated metastasis, was 
admitted to the hospital for a soft tissue tumoral lesion left to midline of the abdomen. Clinical examination revealed a $5 \mathrm{~cm}$ diameter lesion. An elective surgery was performed and the tumoral aspect lesion was resect along with the affected peritoneum, resulting in a full thickness defect of the abdominal wall. The closing technique included the placement of a dual mesh for fascia repair and anatomic suture of the remaining layers of the abdominal wall. The histopathological aspect was suggestive for the diagnosis of liponecrosis with adjacent musculo-aponeurotic inflammatory area, therefore the lesion had a reactive nature, although the aspect was tumoral (figure 6).

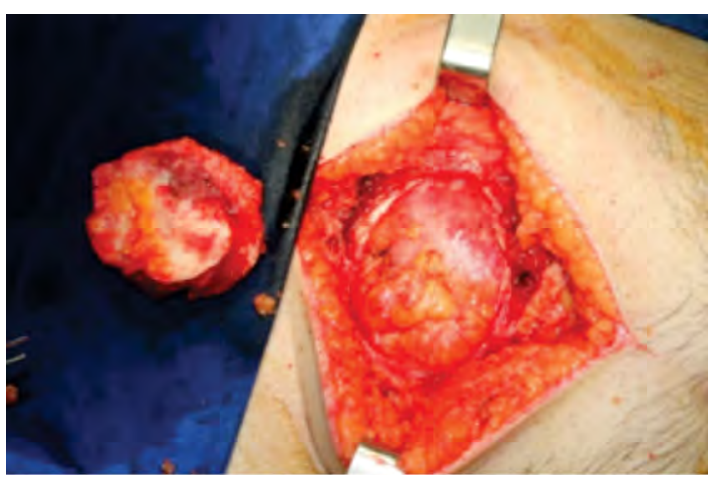

FIGURE 6. Case 4

\section{Case 5}

A 30 years old man was admitted electively to hospital with a large median abdominal eventration. The patient suffered a severe $75 \%$ body sur- face burn injury 3 months before, with a concomitant exploratory laparotomy due to gastroenteric complications. Clinical examination revealed important prolabation of viscera with Valsalva maneuvre. An elective abdominal wall reconstruction was performed using a bilateral anterior component separation technique and reinforcement of the abdominal wall with an onlay polypropylene mesh. The evolution was favorable and the patient was discharged after 7 days with good further recovery (figure 7).

\section{DISCUSSION}

Abdominal wall reconstructive techniques address a wide variety of pathologies including hernia repair, defects resulting after tumor resection, congenital anomalies, and trauma $[7,8]$ The main surgical goals are the following: coverage of the viscera, restoration of the musculo-fascial layers integrity in order to obtain a strong and dynamic abdominall wall and finally achieve an acceptable aesthetic result $[9,10]$.

We further discuss the clinical cases with their clinical particularities and applied therapeutic strategy and try to correlate our data with the existing literature recommendations.

Case 1 - There are many risk factors associated with high risk of wound complications for patients with complex abdomial wall defects, such as obesity, malnutrition, diabetus mellitus, wound infec-
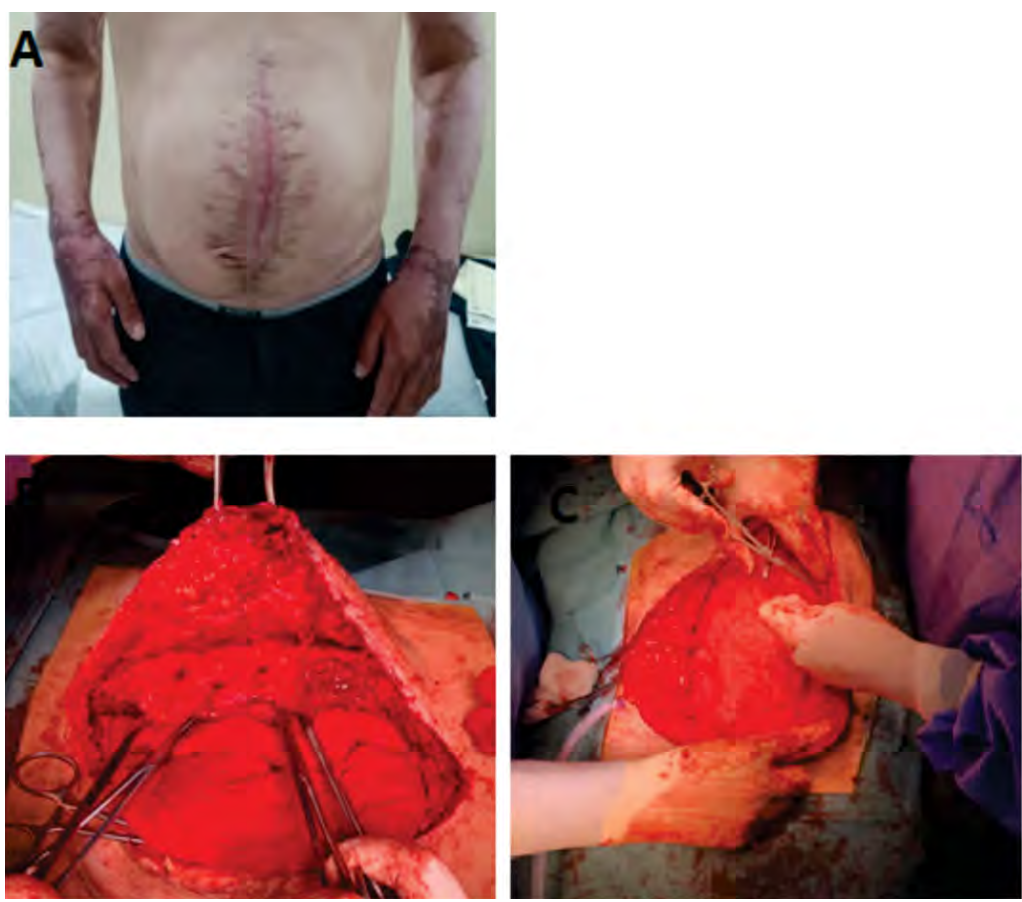

FIGURE 7. Case 5 - A. Aspect before abdominal wall reconstruction;

$B$. Component separation technique; $C$. Onlay mesh placement 
tion, increased dimensions of the defect, smoking, chronic respiratory disease, poor or absent wound healing and failure to reconstruct with mesh or multiple abdominal surgical interventions [2,3]. We notice that our first patient of the case series had three important risk factors: obesity, hypertension, wound infection and multiple surgical interventions. The large defects are difficult to repair directly, due to lack of enough soft tissue available locally, but in our patient, the fascial defect was small enough $(5 \mathrm{~cm})$ and the excess of the surrounding skin and subcutaneous tissue allowed direct closure of the wound. One important aspect is also the long period of hospitalization, 48 days, that involves high costs of the treatment.

Current international guidelines advocate for a preoperative optimization of the general status of the patients requiring abdominal wall repair. Prehabilitation involve adressing specific risk factors, from which smoking cessation, thourogh diabetes control and weight loss are the most important strategies. Other important aspects are chronic infection control, exercise program for debilitated patient, chronic pain management, withdraw substance and alcohol consumption and treatment of chronic diseases (cardiac, respiratory, renal, metabolic) with potential outcome on patient therapeutic response [11-13].

Case 2 - Sometimes, in emergency, the complete medical history of the patient cannot be obtained like in this case, where intraoperative, an infected mesh was found and most probably was the source of wound complications. The risk factors involved here are also obesity, multiple previous surgical interventions on the abdomen, wound infection and not ultimately a failed attempt to reconstruct the abdominal wall with mesh. Usually, for central myofascial defects, using the bilateral component separation technique allow the advancement of the rectus abdominis muscles on the midline and the placement of a mesh to reinforce the abdominal wall [14]. Our patient would have been a candidate for this technique, regarding that CT scan showed a $20 \mathrm{~cm}$ diameter defect, but being an infected wound, the best option was first to remove the mesh and convert the wound to a clean one using a negative pressure wound therapy. It proved to be a good option as the wound was clean and suitable for skin grafting after 14 days (and further performing a more complex planned reconstructive procedure), but unfortunately the patient died due to systemic complications.

Negative pressure therapy (NP) has entered into therapeutic repertoire of many conditions including abdominal wall pathology. The benefits of NP include: decreases the dimensions of the lesions, decrease of bacterial load, stimulate healing process and also reduces the frequency of changing the dressings with consecutive cumulative cost reduction and more comfort for the patient [15]. This approach is very useful for a temporization of definitive coverage in difficult cases with improved reconstructive outcome [14].

Case 3 - This patient had no notifiable risk factors for wound complications before suffering the polytrauma. Because of the high energy of the injury, the soft tissues of the abdominal wall have continuously became necrotic and required multiple debridement. Sometimes, even if all efforts must be done to close the fascia, this is not possible due to extensive resection of soft tissues. Leaving the abdomen open has proven to be an effective treatment for severe abdominal injury, especially when multiple interventions are planned $[16,17]$. This was the therapeutic option for this patient, in order to temporize him for a reconstructive surgery. This patient would have been an ideal candidate for a microsurgical reconstruction with an antero-lateral thigh flap or tensor fascia lata flap, regarding that this technique is best performed on full thickness defects that comprise the superior third of the abdominal wall $[9,14]$.

An emerging surgical technique is abdominal wall vascularized composite allotransplantation. For now, this therapeutic option is not used as independent procedure, but in association with small bowel transplantation or multi-organ transplants, when it is impossible to close the abdominal wall post-transplant, noting that these patients already require immunosuppressive medication for a life-saving transplantation [18-20]. The use of abdominal wall allotransplantation as an isolated reconstructive procedure will be possible in the future only in case of the development of safer immunosuppressive protocols with minimal adverse reactions or promoting specific strategies to induce immunological tolerance [21].

Case 4 - The patient had a tumoral pathology, therefore, the main goal of the surgery was to remove the tumor-like lesion with safety borders and efficiently repair the abdominal wall. Considering that the patient had metastatic lung cancer, we expected that the lesion was also malignant. Although in our case, the hystopathological examination revealed a reactive nature of the lesion, intraoperatively, the necessity to resect the affected peritoneum led to a full-thickness defect of the abdominal wall. It has been proven that using a mesh to rein- 
force the abdominal wall even the direct suture of the fascia is possible decreases the risk of reccurrence with $50 \%$ and this is the reason why we chose to reinforce the direct fascial repair with a dual mesh [22].

Case 5 - This young patient suffered extensive burns and during hospitalization developed digestive complication requiring exploratory laparotomy. Being a severely burned patient, the systemic metabolic and nutritional status was severely impaired, adversely affecting wound healing at the level of surgery performed on the abdominal wall, therefore resulting a parietal complex defect (adressed after improvement of general status of the patient). Bilateral anterior component separation was the elected option, being a reliable technique which allows a functional abdominal wall reconstruction, avoiding a second donor site. The use onlay synthetic mesh is useful to reinforce the weak areas with potential of hernia formation, preventing further complications [14].

Postoperative adequate care is mandatory for patients following abdominal wall reconstructive surgery; besides specific local recommendations, education of the patient regarding important health aspects is mandatory, including promoting early ambulation, exercise program at home as well as in a rehabilitation center, proper nutrition and thor- ough control of other medical comorbidities [23]. Long-term follow-up is essential for ensure functional recovery and durable results.

\section{CONCLUSIONS}

Treating complex abdominal wall defects is challenging and technically demanding, requiring a multidisciplinary collaboration. An important aspect is to obtain a preoperative systematic assessment of the patient with comprehensive workup of risk factors, metabolic status, analysis of previous reconstructive failure, thorough evaluation of actual defect in order to select best surgical strategy. This approach is ideal and can be applied to planned reconstruction, but it is difficult to follow the same steps in emergency cases. The emergency surgeries are focused on treating visceral complications and reconstruction of the abdominal defect is temporized in most cases if the patients survive. There is no standard protocol to treat these patients and choosing the right therapeutic option can have many pitfalls. Therefore, an individualized treatment is necessary, and we find this as an urge for a treatment algorithm in order to promote functional recovery and decrease postoperative complications.

Conflict of interest: none declared Financial support: none declared

\section{REFERENCES}

1. Kawamura K, Luna ICG, Anlicoara R, Lopes PS, Lima MFMB. Reconstruction of the abdominal wall: A case series. Rev. Bras. Cir. Plast. 2018;33(1):56-63

2. Piccoli $M$, Agresta F, Attinà GM, Amabile $D$, Marchi $D$; "Complex abdominal wall study" Italian Collaborative Group. "Complex abdominal wall" management: Evidence-based guidelines of the Italian Consensus Conference. Updates Surg. 2019;71(2):255-272.

3. Khansa I, Janis JE. The 4 Principles of Complex Abdominal Wall Reconstruction. PlastReconstr Surg Glob Open. 2019;7(12):e2549.

4. Hope WW, Abdul W, Winters R. Abdominal Wall Reconstruction. In: StatPearls Treasure Island (FL): StatPearls Publishing; 2020.

5. Disa JJ, Goldberg NH, Carlton JM, Robertson BC, Slezak S. Restoring abdominal wall integrity in contaminated tissue-deficient wounds using autologous fascia grafts. PlastReconstr Surg. 1998;101(4):979-986.

6. DeNoto III G, Reaven N, Funk S, Willis S. Ventral hernia: Retrospective cost analysis of primary repair, repair with synthetic mesh, and repair with acellular xenograft implant. Open Access Surgery. 2013;6:23-32.

7. Bath AS, Patnaik PK, Bhandari PS. Reconstruction of Complex Abdominal Wall Defects. Med J Armed Forces India. 2007;63(2):123126.

8. Mathes SJ, Steinwald PM, Foster RD, Hoffman WY, Anthony JP. Complex abdominal wall reconstruction: A comparison of flap and mesh closure. Ann Surg. 2000;232(4):586-596.

9. Sacks JM, Broyles JM, Baumann DP. Considerations in abdominal wall reconstruction. Semin Plast Surg. 2012;26(1):5-7.
10. 10. Ghazi B, Deigni O, Yezhelyev M, Losken A. Current options in the management of complex abdominal wall defects. Ann Plast Surg. 2011;66(5):488-492.

11. Joslyn NA, Esmonde NO, Martindale RG, Hansen J, Khansa I, Janis JE. Evidence-Based Strategies for the Prehabilitation of the Abdominal Wall Reconstruction Patient. PlastReconstr Surg. 2018;142(3 Suppl):21S-29S.

12. 1 Petro CC, Prabhu AS. Preoperative Planning and Patient Optimization. Surg Clin North Am. 2018;98(3):483-497.

13. Knapp N, Jedrzejewski B, Martindale R. Abdominal wall procedures: The benefits of prehabilitation. PlastAesthet Res. 2020;7:7.

14. Patel NG, Ratanshi I, Buchel EW. The Best of Abdominal Wall Reconstruction. PlastReconstr Surg. 2018;141(1):113e-136e.

15. Tadisina KK, Chopra K, Sabino J et al. Negative pressure wound therapy for abdominal wall reconstruction. Eplasty. 2013;13:ic60.

16. Huang Q, Li J, Lau WY. Techniques for Abdominal Wall Closure after Damage Control Laparotomy: From Temporary Abdominal Closure to Early/Delayed Fascial Closure - A Review. Gastroenterol Res Pract. 2016;2016:2073260.

17. Coccolini $F$, Roberts $D$, Ansaloni $L$ et al. The open abdomen in trauma and non-trauma patients: WSES guidelines. World J Emerg Surg. 2018;13:7.

18. Levi DM, Tzakis AG, Kato T et al. Transplantation of the abdominal wall. Lancet. 2003;361:2173.

19. Berli JU, Broyles JM, Lough D, Shridharani SM, Rochlin D, Cooney DS, Lee WP, Brandacher G, Sacks JM. Current concepts, and 
systematic review of vascularized composite allotransplantation of the abdominal wall. Clin Transplant. 2013;27(6):781-9.

20. Avashia YJ, Mackert GA, May B, Erdmann D, Ravindra KV. Abdominal Wall Transplantation. Curr Transplant Rep. 2015;2:269-75.

21. Ruiz P, Maldonado P, Hidalgo Y et al. Transplant tolerance: New insights and strategies for long-term allograft acceptance. Clin Dev Immunol. 2013;2013:210506.
22. Luijendijk RW, Hop WC, van den Tol MP et al. A comparison of suture repair with mesh repair for incisional hernia. N Engl J Med. 2000;343(6):392-398.

23. Nahabedian MY, Nahabedian AG. Abdominal wall reconstruction: Strategies to enhance outcomes. OR Nurse 2015; 9(6):14-21. 